\title{
Experimental Identification of Evaporator Dynamics for Vapor Compression Refrigeration Cycle During Phase Transition
}

\author{
Juan E. Catano, TieJun Zhang, Yoav Peles, Michael K. Jensen, John T. Wen \\ Center for Automation Technologies and Systems, \\ Department of Mechanical, Aerospace, \& Nuclear Engineering, \\ Department of Electrical, Computer, \& Systems Engineering, \\ Rensselaer Polytechnic Institute, 110 8th Street, Troy, NY 12180, USA \\ Email: $\{$ catanj, zhangt6, pelesy, jensem, wenj\}@ rpi.edu
}

\begin{abstract}
Experimental identification of the evaporator dynamics in a vapor compression cycle (VCC) subjected to imposed heat flux in the evaporator is studied. The imposed heat flux boundary condition represents a specific application of the VCC for electronics cooling. However, this application requires different models and control algorithms than traditional VCCs with fluid-to-fluid heat exchanges because of the faster time response of the imposed heat flux boundary condition and because the refrigerant flow at the exit of the evaporator is expected to be a two-phase mixture. First principle models are highly nonlinear and, hence, are not practical for system control. To obtain a simplified model, a dynamic identification of component response characteristics is performed by applying a pulse change in the system operating conditions (i.e., the heat load to the evaporator, the expansion valve opening, or the compressor speed). It is shown that for changes in expansion valve opening, the temperature of the refrigerant at the exit of the evaporator has opposite trends when the flow is initially in the two-phase region and when the flow is in the superheated region. This phenomena represents a challenge for controller design, and further investigation is required.
\end{abstract}

\section{Introduction}

The increased power density of electronic components has made traditional cooling methods, like conventional air cooled heat sinks, inadequate ([4]). The need to remove high heat fluxes while maintaining electronic components at relatively low temperatures has made vapor compression refrigeration cycles (VCC) a promising alternative for electronics cooling ([3], [5]). Furthermore, VCCs can be used to cool multiple independent heat sources using the same main refrigeration loop ([6]), making it advantageous for applications like data centers or modern military ships and aircrafts where weapons, sensors, signal processing systems, and computers need to be cooled to maintain optimal performance. However, as mentioned by [1], system-level cooling issues like design, optimization, and control of two-phase systems are some of the major research needs in thermal management of military, automotive, and harsh-environment electronic systems.
The use of VCCs for electronics cooling brings certain concerns that differ from traditional VCCs such as the critical heat flux (CHF) condition in the evaporators and large changes in operating conditions between off-peak performance and full power operation (large transients) which can take place very rapidly. These differences between traditional VCCs and VCCs for electronics cooling need to be addressed with new modeling and control tools. At Rensselaer, simulation models for steady-state optimization ([7], [8]), transient analysis ([9]), and control algorithms for VCC, in electronics cooling are currently being developed.

A schematic of a VCC for electronics cooling is presented in Figure 1. Because of the CHF concern, superheated flow at the exit of the evaporator is an undesirable condition. By allowing two-phase flow to exit the evaporator a heated accumulator is necessary to ensure the safe operation of the compressor by supplying saturated or superheated vapor to the compressor. Nevertheless, transitions between two-phase and superheated regions may occur during large transients, and the control system must be able to handle these transitions. However, these phase transitions represent a challenge for controller design because a switch in the steady-state gain sign can occur in the refrigerant temperature at the exit of the evaporator. This issue can be addressed with proper modeling and control of the system.
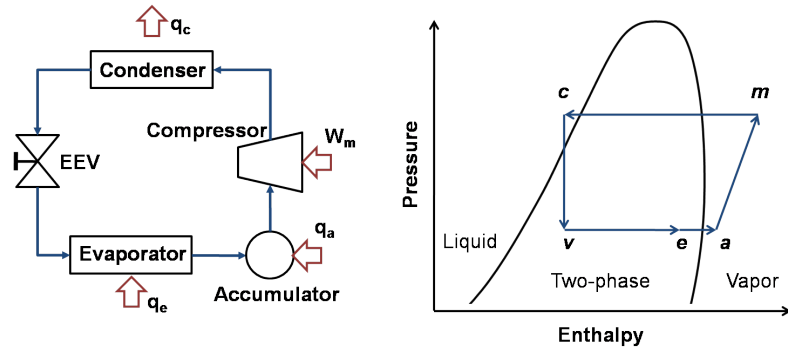

Fig. 1. Schematic of Vapor Compression Cycle

First principle models are highly nonlinear and need to be simplified to be practical for VCC control ([10], [2]). Experimental identification of system dynamics is required for model validation and can also be used for simple controller design. In this paper, experimental identification of a 
VCC dynamics is obtained by measuring the system response to pulse changes in operating conditions (expansion valve opening, compressor speed, or evaporator heat load). The pulse changes are given from a steady-state condition and measurements are taken until steady-state is again reached after the disturbance. Experiments are performed at two different regimes: Where the initial steady-state flow at the exit of the evaporator is in the two-phase region and there is no transition during the disturbance; and where the pulse causes the flow to transition from the two-phase into the superheated region.

\section{Experimental Set-up}

Figure 2 depicts a schematic of the experimental set-up of the VCC with imposed heat flux in the evaporators. It consists of three cartridge heaters immersed in the refrigeration loop which act as evaporators, an electronic expansion valve for each evaporator, a heated accumulator, which allows for low quality at the evaporators outlets, necessary for safe operation of compressor while avoiding CHF conditions, two compressors, a variable frequency drive (VFD), a condenser, and a subcooler, which is connected to a chiller. The testbed is instrumented with three absolute pressure transducers, five differential pressure transducers, 16 type $\mathrm{T}$ thermocouples, and four Coriolis mass flow sensors. The sensors are placed to obtain absolute pressure and temperature at the inlet and outlet of each main component (compressor, condenser, expansion valves, and evaporators).

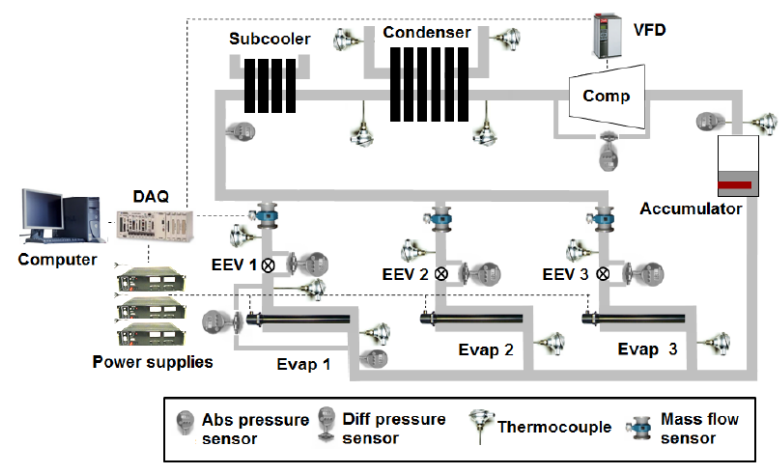

Fig. 2. Schematic of experimental Set-up

The controllable parameters are the compressor frequency $(\omega)$, expansion valve opening $\left(A_{v}\right)$, and evaporator heat load $\left(q_{e}\right)$, which are controlled from a computer using LabVIEW and National Instruments input and output modules. In addition the mass flow rate of the cooling water in the condenser and the temperature of the chiller can be manually regulated. The use of either of the two compressors allows for a large range of operating conditions from $0.8 \mathrm{~kW}$ (small compressor) to $6 \mathrm{~kW}$ (medium compressor). Each evaporator can input up to $2.5 \mathrm{~kW}$. The accuracy of the temperature, pressure, and mass flow rate are $\pm 0.3^{\circ} \mathrm{C}, \pm 0.3 \%, \pm 2 \%$, respectively.

\section{Dynamic Identification of Evaporator Outer Fluid Temperature}

For a constant heat flux device, the maximum wall temperature will occur at the exit of the device. Hence, for the system dynamics identification, the fluid temperature at the evaporator exit $\left(T_{e}\right)$ is selected as the variable of interest. $T_{e}$ is directly related to the temperature of the electronic components through an energy balance on the heater wall (Eq. 1):

$$
c_{p_{-} w} \rho_{w} V_{w} \frac{\partial T_{w}}{\partial t}=q_{e}-\alpha_{e} A_{e}\left(T_{w}-T_{e}\right)
$$

For a fix evaporator and heater geometry and heat capacity, the only method to control the temperature of the electronic component $\left(T_{w}\right)$ when the heat load is changed is by controlling $T_{e}$ or the heat transfer coefficient $\left(\alpha_{e}\right)$. The heat transfer coefficient cannot be measured directly and for two-phase flow is correlated using variables like mass flow rate, flow quality, and fluid properties. Therefore, the current analysis focuses on the dynamics of $T_{e}$. Future work will include determining the best correlation for the calculation of the heat transfer coefficient in the current experimental system and obtaining the dynamics of $T_{w}$.

To experimentally identify the evaporator dynamics incremental and decremental pulse changes are introduced to the VCC operating conditions $\left(A_{v}, \omega\right.$, and $\left.q_{e}\right) . A_{v}$ and $\omega$ are the controlable actuators in the system (manipulable inputs) and $q_{e}$ is the heat load that is determined by the system demand (unmanipulable input). A set of experiments consists of measuring the evaporator outlet fluid temperature at steady-state condition, followed by a pulse in $A_{v}, \omega$, or $q_{e}$, and during its return to steady-state condition. Two sets of experiments were performed for pulses in $A_{v}, \omega$, and $q_{e}$, one for two-phase flow at the evaporator exit during the entire disturbance and the second one for a transition to superheated vapor at the evaporator exit during the pulse. A Matlab code was used to track the dynamic response to these disturbances by fitting a first order and second order model to the measured data. A transfer function relating $T_{e}$ to manipulable and unmanipulable inputs (Eq. 2) is obtained in each case.

$$
Y(s)=G(s) X(s)
$$

\section{Results and Discussion}

Figure 3 shows the results for pulses in $q_{e}$ without phase transition. When $q_{e}$ is increased the pressure in the evaporator raises; hence, the refrigerant temperature initially rises, then the system returns to the original steady-state. From the identification procedure it is clear that the secondorder model matches the system dynamics closer to the measured system than the first-order model. The secondmodel describes the coupling of the heat conduction through the evaporator wall to the heat convection to the refrigerant flow. The transfer function for this case is presented in Eq. 3. 

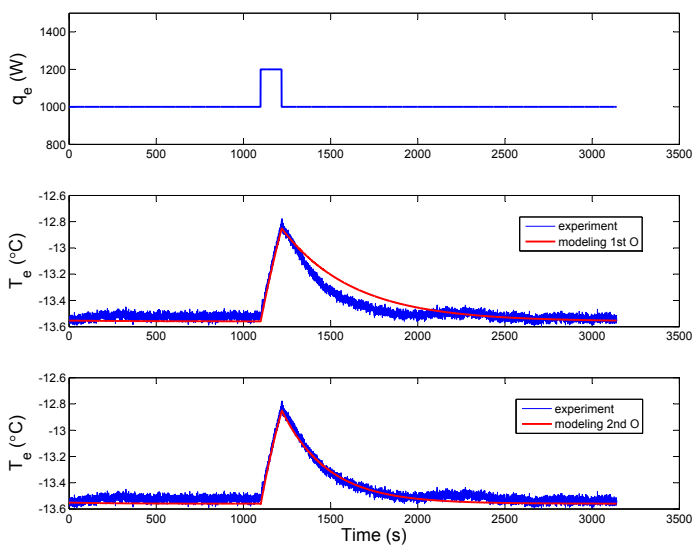

Fig. 3. Evaporator outlet fluid temperature dynamics for a pulse actuation on heat load without phase transition during the pulse

$$
\frac{T_{e}(s)}{q_{e}(s)}=\frac{1.035 e-4+1.107 e-4}{s^{2}+3.023 s+1.142 e-2}
$$

The results from a pulse in $\omega$ with no phase transition are presented in Figure 4. It can be seen that an increase in $\omega$ causes the refrigerant temperature to decrease due to the increase in mass flow rate and pressure drop across the expansion valve. The dynamics can be modeled using a firstorder model because the change in $\omega$ immediately affects the refrigerant mass flow through the evaporator and the pressure level, having a direct effect on the flow temperature at the evaporator exit. The transfer function obtained for this case is presented in Eq. 4.
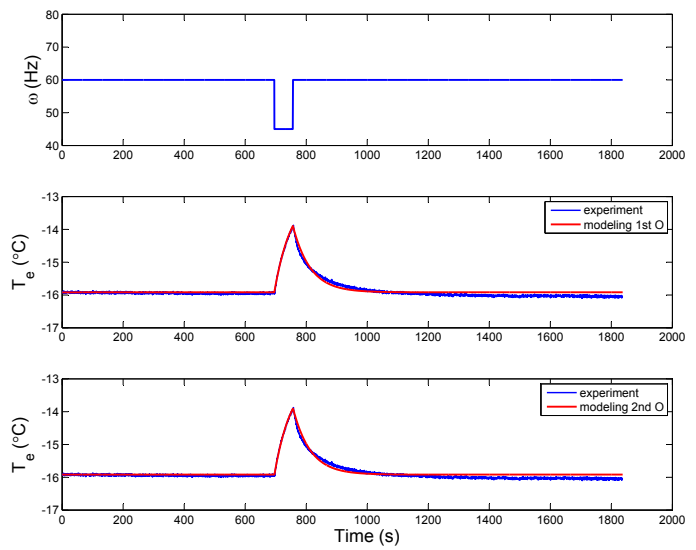

Fig. 4. Evaporator outlet fluid temperature dynamics for a pulse actuation on compressor frequency without phase transition during the pulse

$$
\frac{T_{e}(s)}{\omega(s)}=\frac{-2.023 e-3}{s+1.101 e-2}
$$

In Figure 5, the phase transition is reflected by spikes in the evaporator outlet fluid temperature. For this case the dynamics of the system are very similar to that of the case with no phase transition and if complete transition to superheated region occurs the only effect to the dynamics would be a larger steady-state gain.
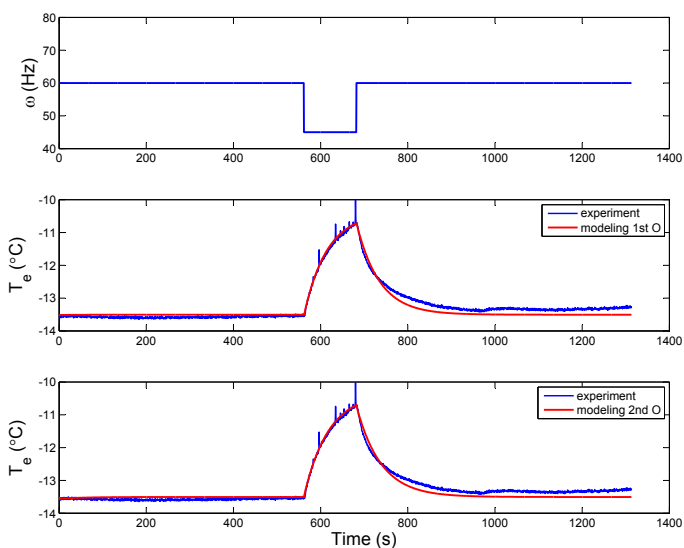

Fig. 5. Evaporator outlet fluid temperature dynamics for a pulse actuation on compressor frequency with refrigerant flow going into the superheated region during the pulse

Figure 6 presents the results for a pulse in $A_{v}$ without transitioning to superheated vapor. For this region an increase in $A_{v}$ is followed by an increase in $T_{e}$ because at higher valve opening the pressure drop across the valve is reduced and the mass flow rate is increased. The increase in mass flow rate guarantees that the flow will remain in the twophase region while the coupling of the saturation pressure with temperature produces the temperature rise. The transfer function for this case is presented in Eq. 5 .
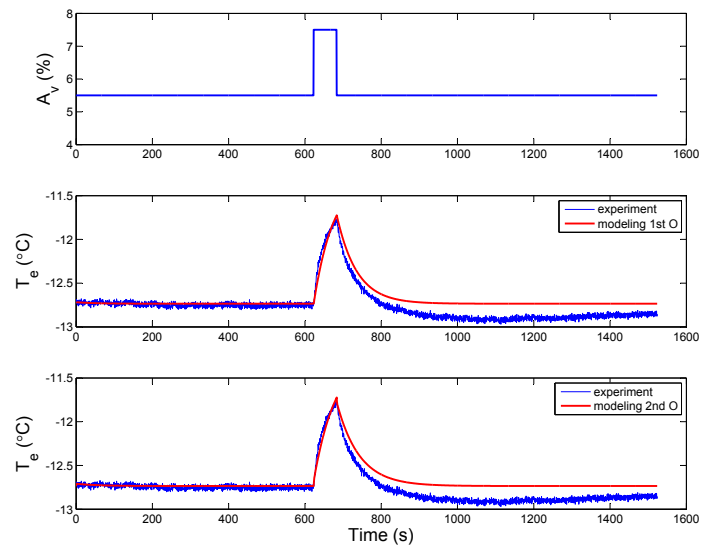

Fig. 6. Evaporator outlet fluid temperature dynamics for a pulse actuation on expansion valve opening without phase transition during the pulse

$$
\frac{T_{e}(s)}{A_{v}}=\frac{1.411 e-2}{s+1.91 e-2}
$$

Figure 7 shows a very interesting phenomena, where by imposing a decremental pulse in $A_{v}$ a significant temperature rise is obtained in $T_{e}$. This means that a rise in the refrigerant 

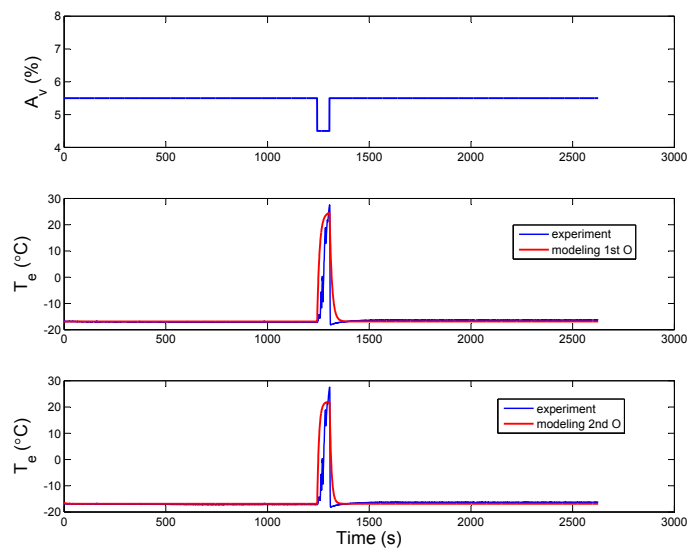

Fig. 7. Evaporator outlet fluid temperature dynamics for a pulse actuation on expansion valve opening with refrigerant flow going into the superheated region during the pulse

temperature can occur for both incremental and decremental pulse in $A_{v}$ depending on the state of the refrigerant flow during the transition. For the transition to the superheated region, the rise in temperature is explained by the decoupling of the temperature and pressure, then for a larger pressure drop the mass flow rate decreases and the energy into the evaporator is balanced by a higher enthalpy and temperature at the exit of the evaporator. The transfer function for the phase transition is presented in Eq. 6

$$
\frac{T_{e}(s)}{A_{v}}=\frac{-3.537}{s+8.507 e-2}
$$

The change in sign for the transfer function for both cases of $A_{v}$ (Eq. 5 and Eq. 6) represents a real challenge for controller design and a traditional PID controller would not be capable of handle this phase transition. Future work includes the development of a methodology for controller design with gain switching.

\section{Conclusions}

The system dynamics for the outlet fluid temperature in the evaporator for a VCC with imposed heat flux as the boundary condition are presented. It has been shown that a first-order model reflects the dynamics of the $T_{e}$ for changes in $\omega$ and $A_{v}$, while disturbances in $q_{e}$ should be modeled using a second-order model. For the cases of varying $\omega$ and $q_{e}$, the dynamics for when the evaporator outlet condition is in the two-phase region or in the superheated region have similar trends but different steady-state gains. However, for the case of varying $A_{v}$ the sign of steady-state gain changes from when the evaporator outlet condition is in the twophase region to when it is in the superheated region, and the same control action on $A_{v}$ can have opposite responses on refrigerant temperature depending on the exit conditions of the flow. The refrigerant temperature at the exit of the evaporator can be related to the compressor frequency using a first-order transfer function with negative gain; an increment in $\omega$ produces a decrease in the refrigerant temperature at the exit of the evaporator. For the case of heat load, the dynamics can be represented by a second-order transfer function with positive gain. The transfer function of $A_{v}$ to refrigerant temperature in the two-phase region can be modeled with a first-order model with positive gain, while for the superheated region the gain becomes negative. The switch in gains signs for the $A_{v}$ model is due to the decoupling of the pressure and temperature outside the two-phase region.

\section{Acknowledgements}

This work is supported in part by the Office of Naval Research (ONR) under the Multidisciplinary University Research Initiative (MURI) Award GG10919 entitled "SystemLevel Approach for Multi-Phase, Nanotechnology-Enhanced Cooling of High-Power Microelectronic Systems," in part by the National Science Foundation (NSF) Smart Lighting Engineering Research Center (EEC-0812056), and in part by the Center for Automation Technologies and Systems (CATS) under a block grant from the New York State Foundation for Science, Technology and Innovation (NYSTAR).

\section{REFERENCES}

[1] S. V. Garimella, A. S. Fleischer, J. Y. Murthy, et al., "Thermal challenges in next-generation electronic systems", IEEE Trans. on Components and Packaging Technologies, vol.31, pp.801-815, 2008.

[2] X. D. He, S. Liu and H. Asada, "Modeling of vapor compression cycles for multivariable feed back control of HVAC systems", Trans. of the ASME: J. of Dynamic Systems, Measurements and Control, vol.32, pp.453-465, 2009.

[3] J. Lee and I. Mudawar, "Low-temperature two-phase microchannel cooling for high-heat-flux thermal management of defense electronics”, IEEE Trans. on Components and Packaging Technologies, vol.32, pp.453-465, 2009.

[4] S. Trutassanawin, E. Groll, S. V. Garimella and L. Cremaschi, "Experimental investigation of a miniature-scale refrigeration system for electronics cooling", IEEE Trans. on Components and Packaging Technologies, vol.29, pp.678-687, 2006.

[5] A. Nnanna, "Application of refrigeration system in electronics cooling", Applied Thermal Engineering, vol.26, pp.18-27, 2006.

[6] J. L. Lin and T. J. Yeh, "Control of multi-evaporator air-conditioning system for flow distribution", Energy Conversion and Managment, vol.50, pp.1529-1541, 2009.

[7] R. Zhou, J. E. Catano, T. Zhang, J. Wen, G. Michna, Y. Peles, and M. $\mathrm{K}$. Jensen,"The steady-state modelling and static system design of a refrigeration system for high heat flux removal", ASME: International Mechanical Engineering Congress and Exposition, 2008.

[8] P. Phelan, Y. Gupta, H. Tyagi, R. Prasher, J. E. Catano, G. Michna, R. Zhou, J. Wen, M. K. Jensen, and Y. Peles "Optimization of refrigeration systems for high-heat-flux microelectronics", ASME: International Mechanical Engineering Congress and Exposition, 2008.

[9] T. Zhang, J. E. Catano, R. Zhou, and J. Wen,"ODynamic modeling of refrigeration cycle for electronics cooling", ASME: International Mechanical Engineering Congress and Exposition, 2008.

[10] B. Rasmussen and A. Alleyne,"Control-orineted modeling of transcritical vapor compression systems", Transactions of the ASME, vol.126, pp.54-64, 2004. 